\title{
Self Organised Group Activities Supported by Asynchronous Structured Conversations
}

\author{
C. Viéville and A. Derycke \\ Laboratoire TRIGONE \\ University of Sciences and Technologies of Lille \\ Cité scientifique \\ 59655 Villeneuve d'ASCQ CEDEX \\ FRANCE \\ email:Claude.Vieville@univ-lille1.fr
}

\begin{abstract}
This paper describes a communication system accessible through a Web browser. The main advantage of this system is to encourage a collaborative way of learning using asynchronous communication channels. The conversation is strongly structured by the system itself which helps the users to co-ordinate their actions playing their respective roles within a task. A conversation always occurs in the context of a task where each user plays a particular role. The system is built around the notion of an active form which is the single way for the user to communicate with the system. This system can be used by the actors of the educative process to self organise their work in a Virtual Campus. The first implementation is now finished and a usability test has been undertaken; the system has been modified in consequence.
\end{abstract}

\section{Keywords}

Asynchronous communication, Coordination, Group activity, Roles, Tasks, Forms, Structured conversation 


\section{INTRODUCTION}

This paper describes an asynchronous collaborative learning system which aims to support a distance education process on the Web. What attracts the Internet to an educational institute is a large communication network to exchange information in two ways, the on-line browser and the courseware package distribution. So the challenge we have to face is to change information exchanges into learning activities. For this reason, we are interested in second generation servers which respond better to educational needs: better interactivity between video-clip, text, images, and so on; enabling re-use of all the supports we have developed in a fully integrated manner; inclusion of graphics and formulae is compulsory for many curricula; embedded courseware corresponds with the multiplicity of training pathways for individualised training and the ease of navigation required. As a minimum requirement, the system needs communication facilities to enhance real collaboration between users and tutors. In the EONT project ${ }^{1}$, in which we are participating, we are verifying these hypotheses. And in the DEMOS project ${ }^{2}$ we are designing, developing the asynchronous communication system presented more precisely in this paper. To develop our system we distinguish three spaces in which the activities of learners take place: information space, action space and communication space. The communication space depends on the institute, and organises the interactivity between the different spaces to correspond to a pedagogical practice. After a short introduction of the application field, the paper presents the functional specification of the system we are currently testing. A participative approach with a user group has been organised and usability metrics of the system are also presented.

Finally in the MODEM project $^{3}$, we are using the potential of ACLS to offer a way to organise the work of the teachers and students in a Virtual Campus. The ACLS is used to determine the scheduling of pedagogical activities, to manage the work to do for the teacher point of view as well as the student point of view. In the framework of this project we conducted a trial which has given us much useful information feedback.

\footnotetext{
1 An experiment in Open Distance Learning using New Technologies - part of the Socrates programme of the European Commission

2 Distance Education and tutoring in heterogeneous teleMatics envirOnmentS - part of the Education and training programme of the European Commission

${ }^{3}$ Multimedia Optimisation and Demonstration for Education in Microelectronics - part of the Education and training programme of the European Commission
} 


\section{THE EDUCATIONAL CONTEXT}

CUEEP (Centre Universite-Economie d'Education Permanente) is an institute of the University of Sciences and Technologies of Lille in northem France which is concerned with several activities: further education for adults, research into educational engineering (open learning and new communication technologies), transfer within the context of new technologies in education.

Since the late 70's, we have introduced new learning technologies and important work has been done in designing and experimenting courseware in traditional situations or in distance education and also in research and development of new tools with the help of European programmes like DELTA where a system of cooperative learning (the Co-Learn project) has been designed and experimented.

Since the late 80's, we have set up an open and distance system mixing several modes of training (group, individualised, distance, self-training in resource). At the moment, people who are registered in distance education are principally those who take a specific examination for adults equivalent to the baccalaureate. They learn from multimedia course material (written paper, audio-tape, video-tape, courseware) and they are in contact with a tutor by phone, fax and Minitel.

Some experiments of the co-operative system Co-learn have been set up during these two last years. Now we are looking to integrate this communication sysiem into our distance education organisation.

To continue our work of research into the use of communication tools in distance education we are conducting a project to deliver a course on the Web based on collaborative learning. This project is mainly supported by the European Commission through the Telematics for Education programme. In this framework, we are setting an Asynchronous Collaborative Learning System in the DEMOS project. This system relies on a second generation of Web server (HyperWave from University of Graz - Austria).

\section{THE USER'S DOMAIN}

In this section the user's domain is clarified and the role of each actor in this area is given as precisely as possible to avoid confusion. From the user's point of view, the prototype that is specified in this document is an asynchronous communication system which favours the co-operation among people belonging to a same "social organisation". More precisely, in this document the organisation is a training institution which proposes this communication system in the framework of its distance education services. We focus only on the delivery side of the training institute - the course material production as well as the administrative side are outside the scope of this study. Two roles have been defined to provide an 
interface with both these other activities. People of this organisation have different roles such as:

- teacher: a person who is responsible for all the courses of a given domain proposed by the organisation to the trainee. He/she is the interface with the course material production teams.

- tutor: someone who gives daily help to a well defined set of learners. He/she works under the direction of a teacher.

- trainer: a person who leads a course, also under the guidance of a teacher.

- counsellor: a counsellor builds individual or group learning paths in negotiation with the learners themselves or human resource managers of the customer companies.

- course manager: somebody who is responsible for providing the requirements of tutors, teachers and learners in order to support the training services. This person is an interface with the administrative side of the training organisation.

- learner: someone who is trained by the institution. A learner may work for another "client organisation", but when he/she uses the services provided by the training institute they belong to the same "social organisation".

- human resource manager: a person working for an external organisation who negotiate learning paths with a counsellor.

Figure 1 represents the conceptual graph defining relations between roles and several basic objects of a training organisation. The number of roles portrayed inside a training organisation has been selectively limited as we are only interested in the "delivery side" of the process. Roles such as editors, course material designers, researchers, administrative staff, and many others are not taken into account in the specification of this communication system.

The system needs to be flexible enough to support various pedagogical scenarios. That means that it has to support several communication modes and it also needs to be adaptable, both during the installation phase and even during the operational process. Flexibility of the communication modes between users is one of the major challenges for this kind of system.

This section has described the context in which the communication system will be used. The following gives a description of the services that the system will provide to its users. 


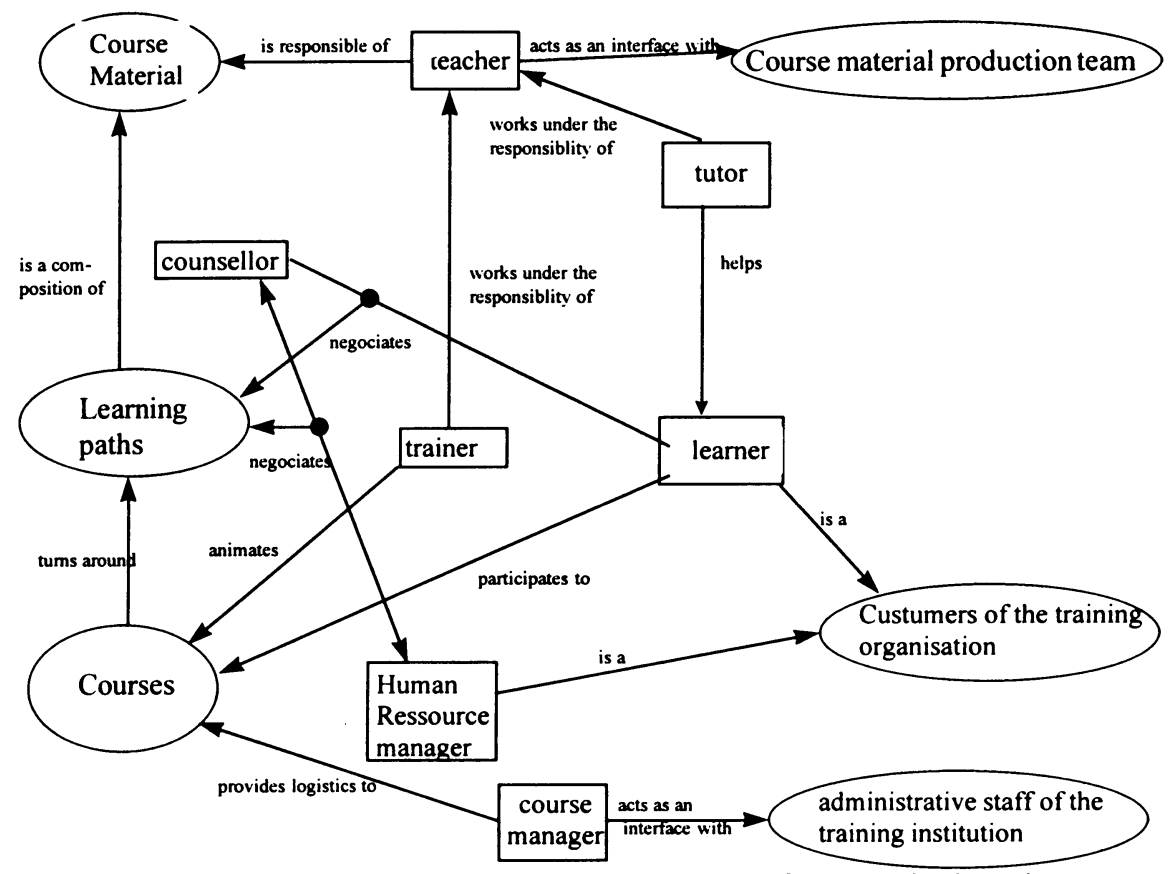

Figure 1. Conceptual graph linking objects of the user's domain

\subsection{Overview of the services}

This asynchronous communication system will provide a set of services from the same family as those already provided by electronic mail (email), electronic forums (forum), Bulletin Board Systems (BBS) and News. Its ambition is to give users real help with their tasks by avoiding several well-known drawbacks of current systems (Terry, 1991) and to propose a structuring of the conversation so that it is very efficient to communicate and collaborate via such a system (Viéville, 1995). The measurement of the efficiency of this system could be made upon :

- time-saved during the co-ordination phase of a collaborative process (Bussler, 1994),

- time-saved when reading each others contributions,

- enhancement of the quality of arguments produced during a debate (Desaranno, 1994),

- better involvement of users in the collaborative processes.

The Co-Learn project is an important input to the specification of such systems. In (Derycke, 1992), the interest of developing Collaborative Learning activities has been explained. It is outside the scope of this document to argue in favour of educational processes which are based on collaboration between learners and tutors. 
In (Kaye, 1995) it is stated as result of the Co-Learn project, that "it might have been preferable to put emphasis on the Asynchronous Communication mode as the basic substrate for communication between learners and tutors. In this way the Asynchronous Communication Mode would provide the glue which would hold a course together, inter-linking the real-time sessions, and providing the forum for continuing discussion and collaboration after each of these sessions." The reader who is interested by this discussion will find pertinent papers on this subject in the reference section (Harasim,1993), (Henri, 1994), (Kirsche, 1994). Jonassen, in (Jonassen, 1996), gives an excellent overview of the possibilities of Computer Mediated Communication (CMC) in educational process.

\subsection{Basic Services}

The ACLS offers a set of basic services enhanced by a subset of complementary services which are needed to manage, adapt and integrate the system using existing communication tools to meet users' needs (Palme, 1992), (Palme, 1993), (Turoff, 1991).

Globally the basic services provided by this asynchronous communication system are:

- informal exchanges between people,

- question-answer exchanges between people,

- date negotiation between people (Woitass, 1990),

- pro-con argument production,

- action negotiation between people (Rogers, 1995),

- opinion collection.

Each of these services could involve people regardless of the context of a collaborative task, or be used in the framework of a task process involving the group. In this latter case the exchange is automatically classed as public, unless specifically defined as private. The task in which the communicators are involved is very fundamental as it will define the context in which the exchange has occurred (Ellis, 1994). In this ACLS electronic mail is not distinguished from electronic forums or news systems as a means of communicating between people. The ACLS provides an integrated view of exchanges whatever channel is used (i.e. email, forums, news, BBS etc.) (Benford, 1992).

This basic service will allow the members to select, fill in, edit, and submit a form which will complete an exchange. Exchanges are linked to each other by a temporal relation. The creation of a new exchange is a particular case of the creation of a contribution which becomes the root of the exchange.

\subsection{Complementary services}

The ACLS also proposes other complementary services to its basic services. To encourage co-operation ACLS will provide a service which gives information on its users. The communication needed by users during the task process will be supported inside a "group activity". The group activity is the context in which the "exchanges" of a communication occur. One and only one organisational group is 
attached to a group activity. The exchanges of a communication are structured sets of contributions. Each exchange is regulated by a set of global rules pre-defined at the installation of the ACLS. This set of rules depends on the way people of the organisation work together (Viéville, 1995). Obviously default rules are proposed during the installation phase. To participate in a group activity a user needs to be added; he then becomes a "member" of the group activity.

It is also possible task by task to create subgroups in which all the members play an identical role with regard to the aim of the task. For example, if a collaborative writing task is started, subgroups of "authors", "editors", "reviewers" are created by the initiator of the task. Belonging to a subgroup will give different rights to the objects in the ACLS.

A search service is available for all the users who want to find any objects in the ACLS. Users, group activities, sub-groups, forms, exchanges and tasks are searched and displayed to the user of the search service. To start a search operation, the user must fill in fields of a search form. The user has to define in the form which criteria the search should use. It is possible to search on the attributes and/or the contents of any kinds of objects of the ACLS.

Authorised users will use the administration service to create/modify attributes; delete/archive/open/close user and group activities. This administration is done by filling in an administrative form.

Users are added and removed from group activities by using the registration service. A subset of authorised users with appropriate rights will have access to this service. Registration is performed by filling out a registration form. Only when a group activity has appropriate parameters may a user register himself for that activity.

A service of notification allows members, who have subscribed, to be notified when something is appended to the group activity. Filling in a notification form is the proposed way to subscribe to the notification service. The notification service allows to the user to receive (or avoid reception of) the events generated inside the ACLS. The kinds of events are :

- "group activity" list has changed,

- list of users of the ACLS has changed,

- status of a group activity has changed,

- list of tasks for a particular group activity has changed,

- list of exchanges for particular tasks has changed,

- list of forms for a particular exchange has changed,

- a deadline relative to a task is going to arrive,

- a deadline relative to a task has been detected,

- a particular user activity has been detected,

- a particular group or subgroup activity has been detected.

The events are sent to the notification recipient which could be an electronic mail address, a news group, or another task of any other group activity. 


\section{SPECIFICATION OF THE USER INTERFACE}

\subsection{Basic principles}

The principle of the user-system dialogue relies on the submission of active forms (Hammainen, 1991). This dialogue implements a schema of information interchange between the user and the system as already exists on the Web with the HTML form. This will remove constraints imposed by the selection of the user's workstation. This choice also allows complete mobility of the user and does not obviates the need to install specific client software on the user's workstation. Within these forms, there are three main objects manipulated by the user inside the ACLS. Another particular object is also manipulated - documents, however these are outside the scope of this study. The ACLS forms are too generic to be considered as an object; they are containers for the main objects presented in this section. The semantics which define articulation between these main objects are summarised in the figure 2 .

Task : to perform a task the organisational group can decompose it into several sub-tasks. The aim of a task is achieved by performing actions external to the system. A task can be personas or collaborative. A task organiser manages the list of tasks by communicating with the mediation agent (mediator). Each group activity has its task organiser.

Exchanges and contribution forms : the contribution form (contribution for short) is the basic element by which users communicate, co-ordinate and work together. A contribution is a semi-structured message (Malone, 1987). It is a container for output and entry fields (list of items, dates, links etc.) and user actions (initiation and commitment of tasks, exchange, validation of forms. etc.). The exchange is the container which structures a set of contributions which appear as a tree-like structure. A mediator manages one and only one exchange; all contribution forms are communicated to the mediator. The mediation agent composes the contribution form according to its status and the role of the communication agent. A contribution is posted with a privacy level.

Documents : Documents are external to the ACLS, but can be attached to main objects of the ACLS. The following rules allows inheritance of attachment through interconnected objects of the ACLS :

- attached documents of a task are also accessible within children tasks

- attached documents of an exchange are also attached within children exchanges and in all the contributions of these exchanges

- attached documents of a group activity are attached to all the tasks and exchanges and contributions

- attached documents of a subgroup are attached to the relative tasks for which this subgroup has been created 


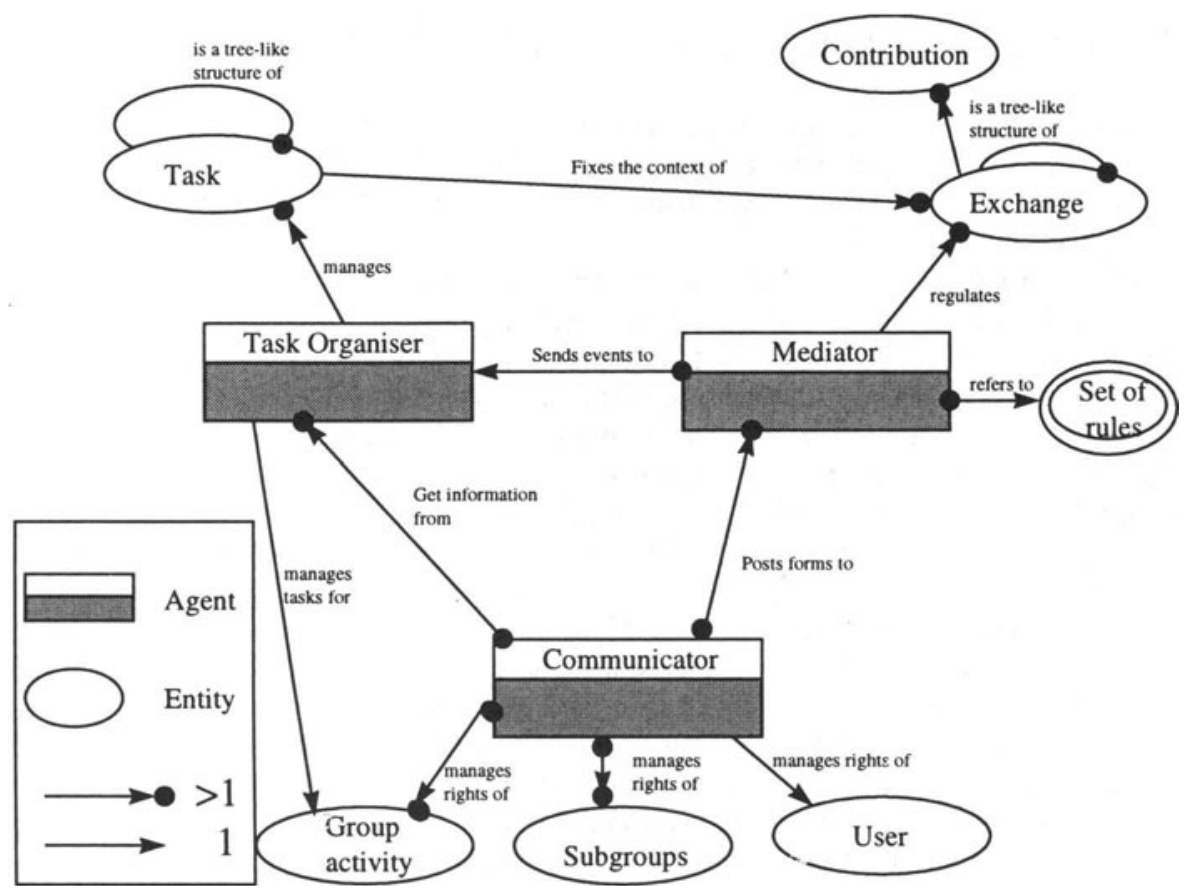

Figure 2. semantic network of basic objects of the ACLS

\subsection{Agents of the ACLS}

In addition to the main objects, there are also agents which "help" the users in their work.

The notification agent (notifier): this entity is responsible for distributing the events which occur in the ACLS to the subscribers who are users of the system. By default the notifier sends mail to signal each event. A notification agent is attached to a social organisation.

Communication agent: Is attached to one user. This agent knows the group activity, the sub-groups each user belongs to and using this knowledge is able to give rights on objects of the ACLS.

The mediation agent (mediator): Organises contributions and in conjunction with the communication agent proposes to the user a well chosen classes of forms to fill in. It also receives forms posted by the communication agent and sends back the form as it will be recorded by the ACLS if the user validates it.

Task organiser: The task organiser manages the users "to-do list". This to-do list is built from events sent by the mediation agent. The task organiser is associated with one and only one organisational group. 


\section{SEVERAL IMPLEMENTATION KEY POINTS}

From the implementation point of view, the Virtual Campus relies on the architecture of an open system of CSCW called ODESCA which is built on the integration of an activity server using an object database for persistency with a WWW information server.

The access to the ACLS functions is realised by the way of the CGI mechanism of a web server. The CGI interface takes in charge the management of the transactions which is not supported by the web servers. This interface is also in charge of the management of the templates database of forms according the organisation and the users. Finally, it also communicates with ODESCA to obtain the conversation state, the list of types of templates allowed for a contribution and other functions less specific to asynchronous communication activities as the information on group members. This CGI application continuously updates a database where the interactions between users and ACLS are stored in order to give information to measure usability of the system.

The data forwarding from the user station and CGI application is done according the HTTP protocol. This protocol does not support a transaction by itself, so a mechanism has been designed to reject a non valid request which has already been submitted. For example, we must avoid a user submitting the same form several times when he uses the moving back functionality of a web browser.

A standard web browser of the Internet allows the user to get the list of the tasks in which he is involved in. Then, using the navigation functionality, he can get the list of the conversations of a selected task. Finally, he will get the list of the contribution of a particular conversation. A synthetic view of the state of the conversation remains always accessible as well as the set of the contents of all the contributions of a conversation (Figure 3 ).

Each time a user wishes to add a contribution at the heart of ACLS, the ODESCA server activates it self to propose him the list if the types of forms which are accessible. This list is computed by taking into account the state of the conversation in which the user wishes to converse, according to the role of the user and according to the kinds of the contributions he has already submitted. For example, in a conversation to define a date, the initiator of this conversation will receive from the ODESCA server a list of two forms: using the first one he will be able to meet the persons at a date selected by the members of the group; with the second one he will be able to announce the abort of the meeting for any reason. The submission of one or the other form will finish the current conversation. In this same conversation, all the other members of this group will receive from ODESCA a form in which he will indicate if the date is convenient for him.

ACLS makes a clear distinction between the presentation objects seen and manipulated by the user and the objects manipulated by it self. When a user creates a new object (i.e. new task, new conversation...) the system selects an appropriate list of templates and the user has then to select one of these. Then, he has to fill the fields of this template. The templates are HTML forms controlled by javascripts. Javascript controls the user input date for each field whose content is interpreted by the system. As the templates are semi-structured messages, some fields are not interpreted by the system but just stored and some other ones needs a strict control. 
the system. As the templates are semi-structured messages, some fields are not interpreted by the system but just stored and some other ones needs a strict control. Before being submitted, a form which carries all the data of the template is locally controlled by a javascript. The designers of these templates encounter difficulties dued to the lack of standardisation of javascript among browsers. Netscape currently presents the most advance features as it is able to manipulate HTML objects such as select object.

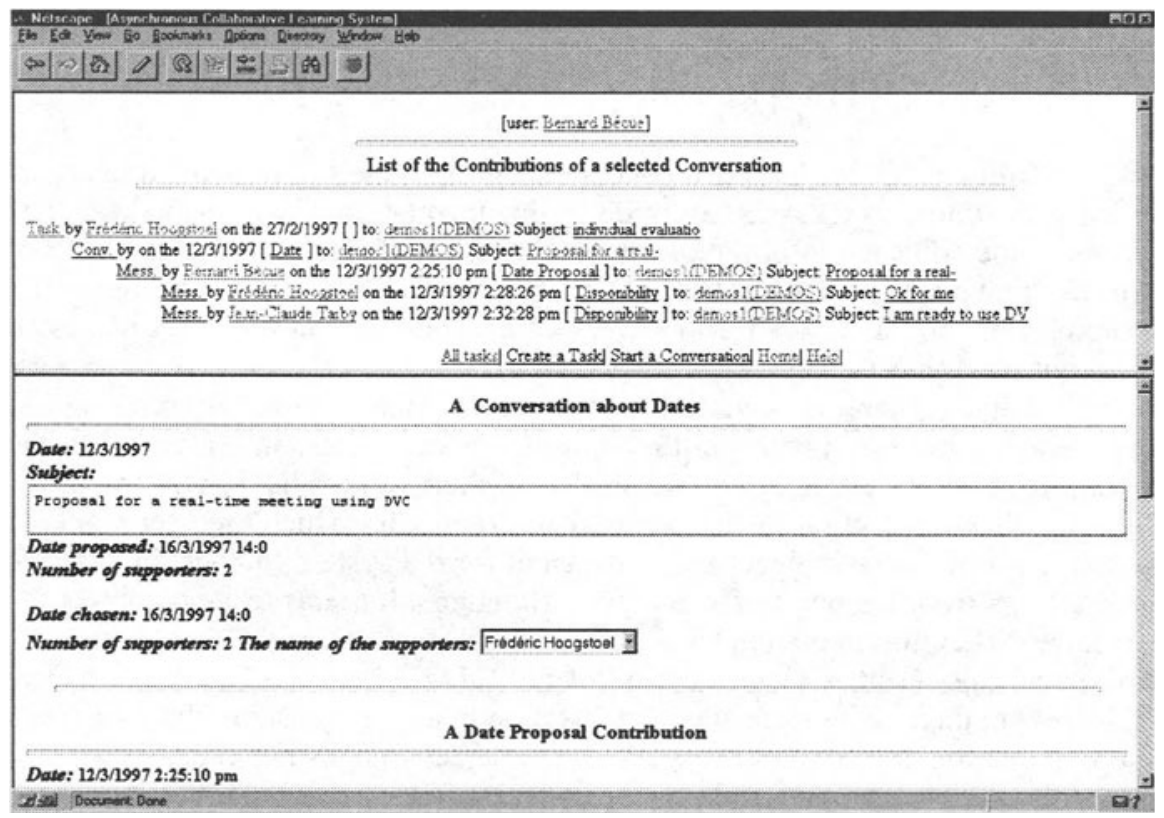

Figure 3. View of a Conversation to agree on a date

The current implementation takes in charge several parameters suitable for the organisations in which ACLS is used but also several other one suitable for the users.

An organisation can select from amongst an existing template database of forms but also edit its own database. The ACLS system uses HTML documents and proposes an extension which allows itself data on the flow according to the state of the conversation or the role of the user. The editing can be done by anybody knowing a HTML editor and the meaning of the variables of the ACLS system. By using a modification process, it is very easy to realise a new templates database in another language. This option is also proposed for use by the user. It can be used to reduce the complexity of a given set of information according to the skill of the users with the system.

As the models are stored in the HTML format, a classic web browser such as Netscape Navigator or Microsoft Internet explorer can be used to access the ACLS system. This choice allows a large usage of the ACLS. 
browser and so more adapted to a specific context of work. The implementation can be done in any language as ACLS interface is just a definition of a protocol. JAVA seems to be a good candidate for this implementation.

The notification mechanism allows users to never consult the ACLS. They only have to let a email agent become active on their station. This agent will receive a notification message coming from ACLS telling them what is new in ACLS for them. A backward link helps them to consult directly the task and the conversation which includes the major events.

\section{FIRST USABILITY TRIALS}

A first usability campaign has been conducted during these last months. It involved ten learners whose work was to write a documentation about a camera. The analysis of the collected information shows a lack of efficiency in the browsing of the tasks, conversations and contributions. The interface designed as a three levels hierarchy needs to be changed. Moreover, we detected that all the users requested help about task objects. They have difficulties in deciding if they have to create a task before starting a new conversation. The result is that they never create a task. They always start conversation in the currently selected task. In consequence the task object never reflects a constextualisation of their work. The only utility of th: task is to allow a selection of the appropriate group with which the user wants to discuss. Even if the task object is fundamental for the system to contextualise the conversations from the point of view of the designers, it seems to be an object the users have difficulties to manipulate.

Following this result, a second design of the HTML forms has been conducted. In this design, the system stores the conversation in a task by asking the user about the group and the objective of his work. If the objective does not exist, the system creates a task to support this new conversation without requesting more information from the user. In consequence, the users are no longer manipulating the «tasks forms ». The system appears simpler. Moreover, the number of levels of objects manipulation (tasks, conversations, contributions) has been decreased by one. This simplification without losing the main advantage of tasks (i.e. contextualisation of work) leads to a more efficient system. People responsible for the implementation of this system inside the organisation have to analyse the work of the users to set up a list of predefined tasks the users will have to conduct. This list can be manually or automatically extended during the live cycle of ACLS. If the user does not find a well suited task in the predefined list he can create a new one. The system administrator can decide to put it in the predefined list if this task seems to be frequently used by the users.

\section{CONCLUSION}

Particular attention has been paid to the methodology of design in order to work with the user group. Its usability has been measured to know how it reduces the coordination times during the self organisation of the asynchronous collaborative 
activities. This system has been designed incrementally; it means that, rapidly, with only a few functions it has been usable by the members of the user group who sent feedback to the designers. This participative approach has certainly given to this system a good level of usability.

\section{REFERENCES}

Benford S., Turroff M. and Palme J. (1992) An ISO standard to support asynchronous group communication. Elsevier Science Publishers B.V. (NorthHolland)

Bussler C. and Joblonski S. (1994) Implementing Agent Coordination for Workflow Management Systems Using Active Database Systems. IEEE software

Danielsen T., Trine Folkow and Per Wiggo Richardson (1988) Relation and Inheritance in Group Communication. Message handling Systems. Elsevier Science Publishers B.V. (North-Holland)

Derycke A. and al (1992) Representation Models for Collaborative Educational Situations and Collaborative Learning activities. Deliverable 4 of Co-Learn D2005

Desaranno S. and Put F. (1994) Co-ordinating group communication. Computer networks and ISDN Systems, Elsevier Science B.V.

Ellis C. and Wainer J. (1994) Goal-based models of collaboration, in Collaborative Computing ( Chapman \& Hall)

Hammainen H. (1991) Form and Room: Metaphors for Groupware. COSC, ACM

Harasim L. (1993) Collaborating in Cyberspace: Using Computer Conferences as a Group Environment. Interactive Learning Environment Vol.3

Henri F. and Rigault C. (1994) Collaborative Distance Learning and Computer Conferencing. NATO ARW, ASI series, Springer Verlag

Hoogstoël F. (1995) Une approche organisationnelle du travail coopératif assisté par ordinateur. Application au projet Co-Learn, thése de Doctorat en Informatique à l'université des Sciences et Technologies de Lille

Jonassen D. (1996) Computers in the classroom; mindtools for critical thinking. Prentice Hall

Kaye A. (1995) Final Evaluation Report, Synthesis of Co-Learn Trials and Experimentation. Deliverable 33 of Co-Learn d2005

Kirsche T., Lenz R., Ruf T., Wedekind H. (1994) Co-operative Problem Solving using Database Conversations. IEEE software

Lindgaard G. (1994) Usability Testing and System Evaluation; a guide for designing useful computer systems. Chapman and Hall

Malone T. (1987) Semi-Structured messages are surprisingly useful for ComputerSupported Collaboration. ACM trans. On Office Information Systems Vol.5

Palme J. (1992) Computer Conferencing Functions and Standards in Collaborative Learning through computer conferencing (Kaye A.R.)

Palme J. (1993) Standards for asynchronous group communication. Computer communication V19 N9, 1993 
Rogers (1995) The Use of an automatic "TO Do " list to Guide Structured Interaction. $\mathrm{CHI} 95$

Smith C. (1994) Co-Learn Usability audit. Deliverable 29a of Co-Learn project D2005

Terry D. (1991) 7 steps to a better mail system. Message handling Systems. Elsevier Science Publishers B.V. (North-Holland)

Turoff M. (1991) Computer-Mediated Communication Requirements for Group Support. Journal of Organizational Computing

Vieville C. (1995) Structuring conversation in asynchronous communication systems to support collaborative learning at a distance. Telematics for Education and training, Held \& Kugemann

Woitass M. (1990) Coordination of Intelligent Office Agents Applied to Meeting Scheduling. Multi-User Interfaces and Applications. Elsevier Science Publishers B.V. (North-Holland) 